\title{
Desvendando o Endereço Físico do Telescópio James Webb
}

\author{
Unveiling the Physical Address of the JWST Telescope
}

\author{
Elaine Cristina Ferreira Silva Fortes ${ }^{* 1}$, Franciane Azevedo ${ }^{2}$, Marcos Kolland ${ }^{1}$ \\ ${ }^{1}$ Universidade Federal do Pampa, Itaqui, RS, Brasil \\ ${ }^{2}$ Universidade Federal do Sul e Sudoeste do Pará, Marabá, PA, Brasil
}

\begin{abstract}
Recebido em 17 de Outubro, 2017. Revisado em 23 de Novembro, 2017. Aceito em 28 de Novembro, 2017.
A localização espacial do futuro telescópio James Webb Satellite Telescope (JWST), em uma posição conhecida como L2 em relação ao sistema Sol-Terra, nos remete ao mais celebrado problema da dinâmica, que é o problema de três corpos. Neste trabalho demonstramos o cálculo do posicionamento do telescópio JWST no espaço, cujo lançamento está previsto para ocorrer em 2019. Para isso, consideramos o problema restrito de três corpos, estudando o movimento de grandes massas se movendo sobre atração gravitacional mútua, considerando neste caso que o terceiro corpo (JWST) tem massa desprezível se comparada aos demais. Além de fazer um breve interlúdio histórico, mostramos as forças envolvidas no cálculo da posição do telescópio JWST no espaço, e fornecemos algumas expressões matemáticas simplificadas para tal ponto de Lagrange. Esse estudo envolve o problema da força central explorado em mecânica, o problema dos pontos de lagrange e suas localizações, explorados em astronomia e em dinâmica do sistema solar e a gravitação clássica de Newton.

Palavras-chave: Problema restrito de três corpos, pontos de Lagrange, Telescópio JWST.
\end{abstract}

The spatial location of the future James Webb Satellite Telescope (JWST), in a position known as the L2 in relation to the Sun-Earth system, direct us to the most celebrated problem in dynamics, which is the three-body problem. In this work we demonstrate the calculation of the JWST position in space, whose launch is expected to occur in 2019. For this purpose, we consider the body restricted problem, studying the movement of large masses moving over mutual gravitational attraction, considering in this case that the third body (JWST) has negligible mass when compared to the others. In addition to making a brief historical interlude, we show the forces involved in calculating the position of the JWST telescope in space and we provide some simplified mathematical expressions for such a Lagrange point. This study involves the problem of central force explored in mechanics, the problem of lagrange points and their locations, explored in astronomy and dynamics of the solar system and Newton's classical gravitation.

Keywords: Three body restrict problem, Lagrangian points, JWST satellite Telescope.

\section{Introdução}

O ano de 2019 promete ser um marco para uma nova era das observações espaciais e o motivo de tanta expectativa deve-se ao lançamento do telescópio espacial JWST (James Webb Space Telescope) previsto para ocorrer entre março e junho desse ano 1]. O JWST foi planejado e construído para ser o grande sucessor dos telescópios espaciais de nova geração, tais como o telescópio Hubble que, foi lançado em abril de 1990 e que em 2017 completou um ciclo de 27 anos marcado por grandes descobertas científicas. O conhecimento mais preciso sobre a idade do universo primitivo, de buracos negros supermassivos, de exoplanetas, do nascimento e de espasmos estelares e ainda sobre a expansão acelerada do universo, devem-se às observações feitas pelo Hubble [2]. Vale destacar também o papel importante desempenhado pelos antecessores deste telescópio, os não menos importantes telescópios Spizer, Keppler, K2 e IRAS 3]. Em se

*Endereço de correspondência: elainefortes@gmail.com tratando de algo mais grandioso, é natural que se criem expectativas ainda maiores sobre o futuro sucessor do Hubble.

O JWST traz consigo muitas características inovadoras. Pode-se afirmar que quando concluído, o JWST terá uma abertura de aproximadamente 6,6 metros de diâmetro, terá o espelho principal constituído por um conjunto de 18 células independentes fabricadas em berílio, totalizando um total de 25 metros quadrados para o registro de raios luminosos 4,5]. E tudo isso para fazer a captação de imagens mais profundas na região do espectro eletromagnético do infravermelho. Por isso sua configuração é bem diferente da configuração de seu antecessor. Para efeitos comparativos, o Hubble contém observatórios orbitais que observam o universo em diferentes comprimentos de onda (luz visível, raios gama, raios-X e o infravermelho). Já no JWST, o sistema óptico infravermelho foi projetado para operar na faixa dos comprimentos da luz laranja ao infravermelho médio. A bordo do observatório estão alocados quatro instrumentos científicos, uma câmera 
de infravermelho-próximo (NIRCam), um espectrógrafo que atua na região do infravermelho-próximo (NIRSpec), um filtro de imagens ajustável (TFI) e um instrumento para análise do espectro na faixa do infravermelho médio (MIRI) [4].

As diferenças entre um e outro telescópio não ocorrem apenas nas configurações. Sabe-se que o telescópio JWST será transportado dos Estados Unidos até a Guiana Francesa. O Hubble encontra-se na órbita terrestre à aproximadamente $589 \mathrm{~km}$, o JWST será lançado pelo veículo espacial Ariane-5 até uma distância de aproximadamente 1,5 milhões de quilômetros da Terra, um ponto conhecido como ponto de Lagrange L2 [4]. Essa posição do telescópio no segundo ponto de Lagrange (L2) é estratégica, e ao contrário do telescópio Hubble, no JWST não está prevista manutenção no espaço. O motivo do lançamento da nave espacial ser localizado na faixa equatorial do planeta (Guiana Francesa), deve-se ao fato da possibilidade de aproveitamento da velocidade de rotação da Terra em sentido favorável à trajetória a ser percorrida pela nave. Esta técnica é conhecida como estilingue gravitacional [6] e suas implicações em missões espaciais são apresentadas na referência 7]. Basicamente no efeito estilingue a energia cinética e momento linear de um corpo são transferidos para um corpo de massa menor, no nosso caso, o veículo espacial Ariane-5, fazendo com que a energia deste veículo cresça de forma prodigiosa.

O objetivo deste trabalho é mostrar o cálculo do posicionamento do telescópio JWST em relação à Terra, nos rementendo ao problema restrito de três corpos, estudando o movimento de grandes massas se movendo sobre atração gravitacional mútua, considerando neste caso que o terceiro corpo (JWST) tem massa desprezível se comparada aos demais. No desenvolvimento deste trabalho, após um breve interlúdio histórico, mostramos as forças envolvidas no cálculo da posição L2 do JWST no espaço, fornecendo expressões matemáticas para a posição tal ponto de Lagrange.

\section{A homenagem a James Edwin Webb e os Objetivos Científicos do Telescópio JWST}

O início século XX foi marcado por um significativo incentivo aos estudos e fomentação de missões espaciais por parte dos EUA. A parte relacionada à pesquisa de questões de observação e instrumentação científica foi estabelecida neste período, com a concepção de uma nova geração dos telescópios espaciais 8 . O nome JWST foi dado em homenagem ao ex-diretor da agência aeronáutica e espacial dos Estados Unidos (NASA), James Edwin Webb (1906-1992). James Webb aceitou a indicação política feita pelo então presidente John F. Kennedy para ser o diretor da NASA. E nessa agência ocupou tal cargo pelo período compreendido entre 1961 e 1969, sendo responsável por conduzir as diretrizes e políticas da NASA. Notoriamente, ele cumpriu a meta estabelecida pelo presidente Kennedy de aterrissar um homem na Lua antes do final da década de 1960. O período compreendido entre essa e outras conquistas ficou conhecido como Era Apollo [9]. James Webb ainda contribuiu para a realização de várias missões espaciais, para a criação do Centro Espacial Johnson, em Houston, para realização de um programa de exploração planetária ( Mariner e Pioneer). E assim em 2002, um telescópio primeiramente batizado como telescópio de nova geração foi renomeado para James Webb Satellite Telescope em homenagem ao segundo diretor da NASA.

As futuras observações do JWST irão gerar informações que darão continuidade às investigações sobre a origem do universo. Os objetivos científicos deste telescópio envolvem a medição das distâncias dos primeiros raios luminosos emitidos após o Big-Bang, o fim da era escura, estudos mais detalhados sobre a matéria escura [4], a evolução do sistema solar, a formação das galáxias, e a busca por sistemas solares que possam ser habitáveis [10]. Algumas características consideradas chaves como resolução espacial, espectral e temporal aumentaram em questões qualitativas, se comparados aos telescópios anteriores como o telescópio Hubble.

\section{O JWST \& Problema Restrito dos Três Corpos na Astronomia}

O problema Newtoniano clássico da interação gravitacional entre corpos celestes e seus movimentos é de extrema importância no contexto da Física e da Astronomia e foi objeto de diversas investigações de grandes físicos, astrônomos e matemáticos por mais de 300 anos [20]. Dentre os notáveis nomes que abordaram e contribuiram significativamente para este problema destacam-se Euler, Lagrange, Laplace, Jacobi, Le Verrier, Hamilton, Poincaré e Birkhoff, Hill entre outros [21. As investigações realizadas por esses cientistas possibilitaram avanços em questões de compreensão de posicionamento de satélites naturais e artificiais, entre outros pontos. Isaac Newton, que formulou a lei da gravitação universal e, posteriormente, desenvolveu preposições a respeito da interação gravitacional entre corpos celestes e seus movimentos (Philosophiæ Naturalis Principia Mathematica, 1687), começou um estudo inicial referente ao problema da interação gravitacional e de movimentação entre três corpos. Ele considerou inicialmente a órbita da Lua em torno da Terra e os efeitos aparentes do Sol nesse movimento. Euler (1722) dando continuidade às investigações, estudou o movimento da Lua, assumindo que a Terra e o Sol orbitavam um centro de massa comum entre ambos, considerando órbitas circulares e tratou a massa da Lua como desprezível em relação às massas do Sol e da Terra. Esta abordagem ficou conhecida como problema restrito dos três corpos. Em paralelo, Joseph Louis Lagrange (1736-1813) descobriu a solução do triângulo colinear e equilateral, sendo esta solução aceita e usada em casos 
de massas arbitrárias em muitos dos problemas atuais relacionados ao tema. Mais tarde, Euler 23 formulou uma solução periódica e relativamente simples sobre o problema dos três corpos. Para isso, ele assumiu como modelo o movimento de elipses colineares, a teoria do movimento dos corpos rígidos (Theory of the Motions of Rigid Bodies, 1765). Em 1836, Jacobi também forneceu importantes contribuições para o problema restrito dos três corpos ao usar um sistema de coordenadas rotacionais, já introduzidas por Euler em 1767 para demonstrar que havia uma integral de movimento do sistema. Essa integral ficou conhecida como integral de Jacobi. Posteriormente a metodologia envolvendo essa integral foi utilizada por George W. Hill (1838-1914) na determinação do movimento de um asteroide no problema de três corpos. Hill ainda introduziu as curvas de velocidades nulas, que estabeleciam as regiões permitidas para o movimento dos corpos celestes e fez um estudo do caso especial onde duas massas eram muito menores se comparadas à uma terceira. Assim, ele descobriu uma nova classe de soluções e dentre suas principais contribuições destacamos uma aproximação para resolver o problema de três corpos envolvendo o sistema Sol-Terra-Lua. Atualmente, essa teoria continua sendo muito usada em mecânica celeste após sofrer algumas modificações.

Pode-se dizer que esse grande período clássico terminou com a proposta de um método mais robusto "surfaces of section, phase space and deterministic chaos", desenvolvido por Poincaré. Vale ressaltar que o trabalho realizado por Poincarè recebeu grande repercusão, pois, até então, os métodos empregados por ele eram incomparavelmente mais profundos e poderosos do que os anteriormente empregados na mecânica celestial e marcaram uma época no desenvolvimento da ciência. Neste trabalho Poincaré desenvolveu métodos para solucionar equações diferenciais e utilizou tais métodos para o estudo de órbitas periódicas, demonstrando ainda a não integrabilidade dos sistemas que descreviam o problema de três corpos. Como bonus, Poincaré conseguir identificar um problema imprevisível e descobriu a primeira manifestação do que hoje é conhecido como caos 22.

Como exemplos possíveis para o problema de três corpos podemos considerar a configuração do Sol-planetaplaneta, Sol-planeta-satélite ou sistemas envolvendo Solplaneta-asteróide. Essa hierarquia de órbitas e massas no nosso sistema solar indica que o problema restrito de três corpos pode fornecer uma aproximação relativamente boa para algumas configurações de massas. Existem variações mais excêntricas para o problema de três corpos tais como o problema de Copenhagen, no qual as duas massas maiores são iguais, e o problema Pitagórico, no qual os três corpos têm massas que obedecem às razões 3:4:5. Neste artigo, estudaremos o caso simples a que se enquadra o posicionamento do telescópio JWST, ou seja, o caso onde a massa de um dos astros é desprezível em relação a massa do outro.

\section{Cálculo da Distância L2 a partir da Terra}

O tratamento feito pela mecânica sobre movimento de dois corpos envolve forças inversalmente proporcionais ao quadrado das distâncias envolvidas e apresenta soluções para as equações de movimento que contemplam órbitas elípticas, parabólicas ou hiperbólicas. Estamos interessados em descobrir o que acontece quando uma terceira massa é adicionada a um sistema como este.

O telescópio JWST pode ser tratado como uma partícula (P) de massa desprezível em movimento, movendo-se sobre a influência gravitacional da Terra e do Sol. Várias soluções podem envolver o problema de três corpos segundo a mecânica. Se por exemplo a energia total desta configuração é positiva, todas as massas podem afastar-se uma das outras, ou ainda uma das partículas pode escapar levando consigo a maior parte da energia, e neste caso, deixando as outras duas para trás descrevendo órbitas elípticas. Se a energia for negativa, uma das partículas pode escapar e deixar as outras duas em um estado ligado, ou ainda as três podem mover-se em órbitas vinculadas sob influência de suas gravidades mútuas. Este último caso se aplica ao telescópio JWST.

O sistema a ser descrito aqui é o problema restrito circular de três corpos, visto que a órbita da Terra em torno do Sol é aproximadamente circular sobre o centro de massa comum entre esses astros. Sendo assim, Sol e Terra irão exercer atração gravitacional sobre o JWST e a influência gravitacional deste telescópio sobre o planeta e o Sol pode ser desconsiderada. Neste trabalho vamos exemplificar o cálculo do posicionamento de um objeto na posição L2 no espaço. Aqui, por simplicidade, tomamos como exemplo o sistema Sol-Terra mas, cálculos semelhantes podem ser feitos para outros sistemas envolvendo outros planetas e seus satélites, etc. Esse cálculo é válido quando a massa de um dos astros é muito menor do que a massa do outro astro, como no caso a ser aqui exemplificado. No cálculo a ser aqui exemplificado usamos a teoria da Gravitação Universal de Newton para descrever a interação entre os três corpos. Outras maneiras de se abordar tal problema poderiam envolver o uso do formalismo Hamiltoniano e ainda do princípio variacional.

Poderíamos nos fazer uma pergunta: Em quais lugares poderíamos estacionar um telescópio, aqui tratado como uma partícula, com uma certa velocidade no referencial inercial, de modo que ele permaneça estacionário em um outro referêncial em movimento de rotação? Para responder a tal pergunta é importante lembrar que em tais lugares, a partícula sofre ação de forças gravitacionais exercidas pelas duas grandes massas e descreve uma órbita kepleriana. Na figura 1, a, b e c denotam respectivamente a localização do Sol, do centro de massa e da Terra com relação ao telescópio, aqui denotado como $\mathrm{P}$. $\mathbf{F}_{1}$ e $\mathbf{F}_{2}$ denotam as forças da partícula por unidade de massa direcionadas para o Sol e a Terra, respectivamente. 


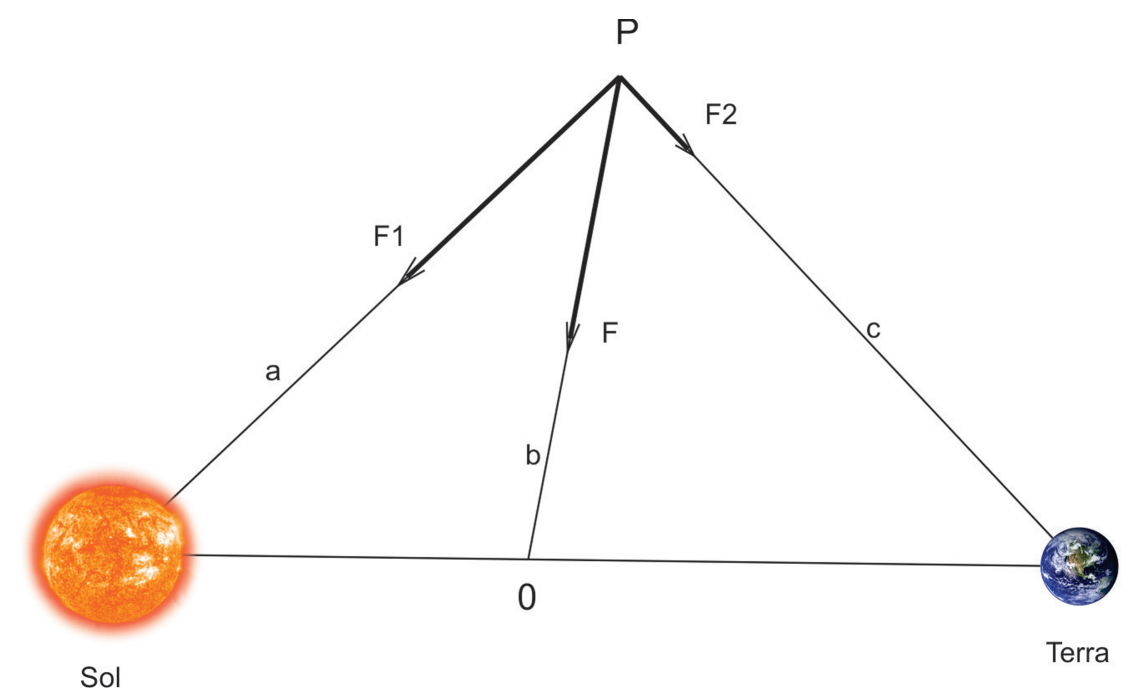

Figura 1: A figura indica as forças exercidas pelas duas massas (Sol e Terra) sobre um objeto aqui denotado por $\mathrm{P}$. $\mathrm{O}$ ponto "O"refere-se à localização do centro de massa entre o Sol e a Terra. As figuras estão fora de escala.

Como a partícula estará estacionada no referencial em rotação, ela deve estar a uma distância fixa b do centro do centro de massa $\mathrm{O}$, sendo $\mathbf{b}$ o único ponto fixo do referencial inercial. Levando em consideração que a partícula move-se numa trajetória circular, ela está sofrendo também a ação de uma força centrípeta, direcionada para -b. Esta força é balançeada pela soma vetorial das forças $\mathbf{F}=\mathbf{F}_{1}+\mathbf{F}_{2}$, dirigida para $\mathbf{b}$ e passando pelo centro de massa $\mathrm{O}$.

Poderíamos também cogitar a existência de uma pseudoforça de Coriolis que poderia ser percebida por uma partícula que está em um referêncial não-inercial em rotação em relação ao referêncial inercial. A força de Coriolis é uma pseudoforça que age em objetos que estão em movimento em relação a um determinado referencial, sendo definida por

$$
F_{\text {Coriolis }}=-2 m_{o b j}\left(\omega \times v_{r}\right),
$$

onde $m_{o b j}$ denota a massa do objeto ou partícula, $v_{r} \mathrm{e}$ $\omega$ denotam respectivamente as velocidades num sistema rotacional e a velocidade angular do objeto em questão. Porém, lembramos que tal pseudoforça não precisa ser levada em consideração nestes cálculos, visto que ela aparece apenas quando a partícula está em movimento em relação ao referencial não-inercial. E este não é o caso do telescópio JWST, visto que ele ocupará uma posição estacionária em relação ao referêncial em rotação.

Para responder à pergunta feita no segundo parágrafo desta seção, faz-se um cálculo matemático envolvendo o balançeamento do vetor força $\mathbf{F}$ em uma direção arbitrária e conclui-se que o sistema tem um ponto de equilíbrio no vértice de um triângulo equilátero, cuja base é formada pela linha que une as massas do Sol e da Terra. Por simetria, sabe-se que existe outro ponto de equilíbrio abaixo da mesma linha sobre o vértice de um triângulo equilátero. Esses pontos estacionários são conhecidos como L4 e L5, respectivamente. Cálculos sub- sequentes indicam a existência de outros três pontos L1, L2 e L3 localizados sobre a linha que une as três massas, conforme pode ser visto na figura 2 Exemplificamos na Tabela 1 objetos naturais e artificiais que ocupam os pontos de Lagrange no sistema solar.

As soluções para a localização dos cinco pontos de equilíbrio (L1, L2, L3, L4 e L5), mencionados no parágrafo anterior, podem ser encontradas, exigindo-se que nesses pontos a partícula tenha velocidade e aceleração nulas no referencial em rotação. Vamos considerar que o movimento da partícula, do Sol e da Terra ocorrem num plano $x-y$. Esta foi uma solução explorada por Euler, onde uma das massas encontrava-se em linha reta entre as outras duas massas envolvendo uma configuração de energia negativa, ou seja, um sistema ligado.

Para o cálculo mais específico do posicionamento do telescópio JWST assume-se que $y=0$ e neste caso há três pontos de equilíbrio L1, L2 e L3 localizados ao longo do eixo x. Estas soluções também são conhecidas como pontos de Lagrange colineares. Neste caso, o JWST ocupárá a posição L2.

Vamos simplificar o cálculo para o posicionamento do JWST no espaço. Seja $M$ a massa do Sol, $m$ a massa da Terra, $r$ a distância da Terra ao Sol, $v$ a velocidade da Terra, $v_{s}$ a velocidade do satélite, $m_{s}$ a massa do satélite localizado na linha que une o Sol à Terra, $T$ o período que a Terra leva para dar a volta em torno do Sol, $R+r$ a distância do satélite que estará posicionado em L2 em relação ao Sol-Terra. O satélite sofrerá influência das forças gravitacionais combinadas $(\mathbf{F})$ exercidas pelo Sol e pela Terra e acompanhará a Terra, descrevendo portanto uma órbita circular em torno do Sol. Escrevendo $F$ em sua forma escalar, temos

$$
F=\frac{G M m_{s}}{(r+R)^{2}}+\frac{G m m_{s}}{R^{2}}
$$


Tabela 1: Exemplos de objetos naturais e artificiais em pontos de Lagrange.

\begin{tabular}{|c|c|c|c|c|}
\hline $\begin{array}{l}\text { Objetos em Pontos } \\
\text { de Lagrange }\end{array}$ & L1 & L2 & L4 & L5 \\
\hline Sol-Terra & $\begin{array}{l}\text { ISEE-3 11], Genesis, } \\
\text { Lisa WIND 12], } \\
\text { SOHO } 13, \\
\text { ACE } 14], \\
\text { DSCOVR 15 }\end{array}$ & $\begin{array}{c}\text { WMAP } 16, \\
\text { Chang'e }[17, \\
\text { Observatórios Espaciais } \\
\text { Herschel e Gaia } 18 .\end{array}$ & $\begin{array}{c}\text { Asteroid } 2010 \\
\mathrm{TK}_{7}, \mathrm{STEREO} \mathrm{A}\end{array}$ & $\begin{array}{c}\text { Asteroid (419624) } 2010 \\
\text { SO }_{16}, \text { STEREO B } \\
\text { Telescópio Spitzer }\end{array}$ \\
\hline Terra-Lua & & $\begin{array}{r}\text { ARTEMIS } \frac{19}{5-\mathrm{T} 1}, \text { Chang' } \mathrm{e} \\
\end{array}$ & Espaçonave Hiter ${ }^{1}$ & Espaçonave Hiten \\
\hline Sol-Venus & & & 2013 ND15 & \\
\hline Sol-Marte & & & $\begin{array}{c}\text { Asteróide (121514) } 1999 \\
\text { UJ7 }\end{array}$ & 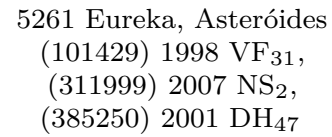 \\
\hline Sol-Júpiter & & & Asteróides Troianos & Asteróides Troianos \\
\hline Saturno - Tétis & & & $\begin{array}{l}\text { Satélite Telesto } \\
\text { Asteróides (385695) } 2005\end{array}$ & Satélite Calipso \\
\hline Sol-Netuno & & & $\begin{array}{c}\mathrm{TO}_{74}, 2001 \mathrm{QR}_{322}, 2005 \\
\mathrm{TN}_{53}, 2006 \mathrm{RJ}_{103}, 2007 \\
\mathrm{VL}_{305}\end{array}$ & $\begin{array}{l}\text { Asteróides } 2008 \mathrm{LC}_{18} \\
2004 \mathrm{KV}_{1} 8,2011 \mathrm{HM}_{102}\end{array}$ \\
\hline
\end{tabular}

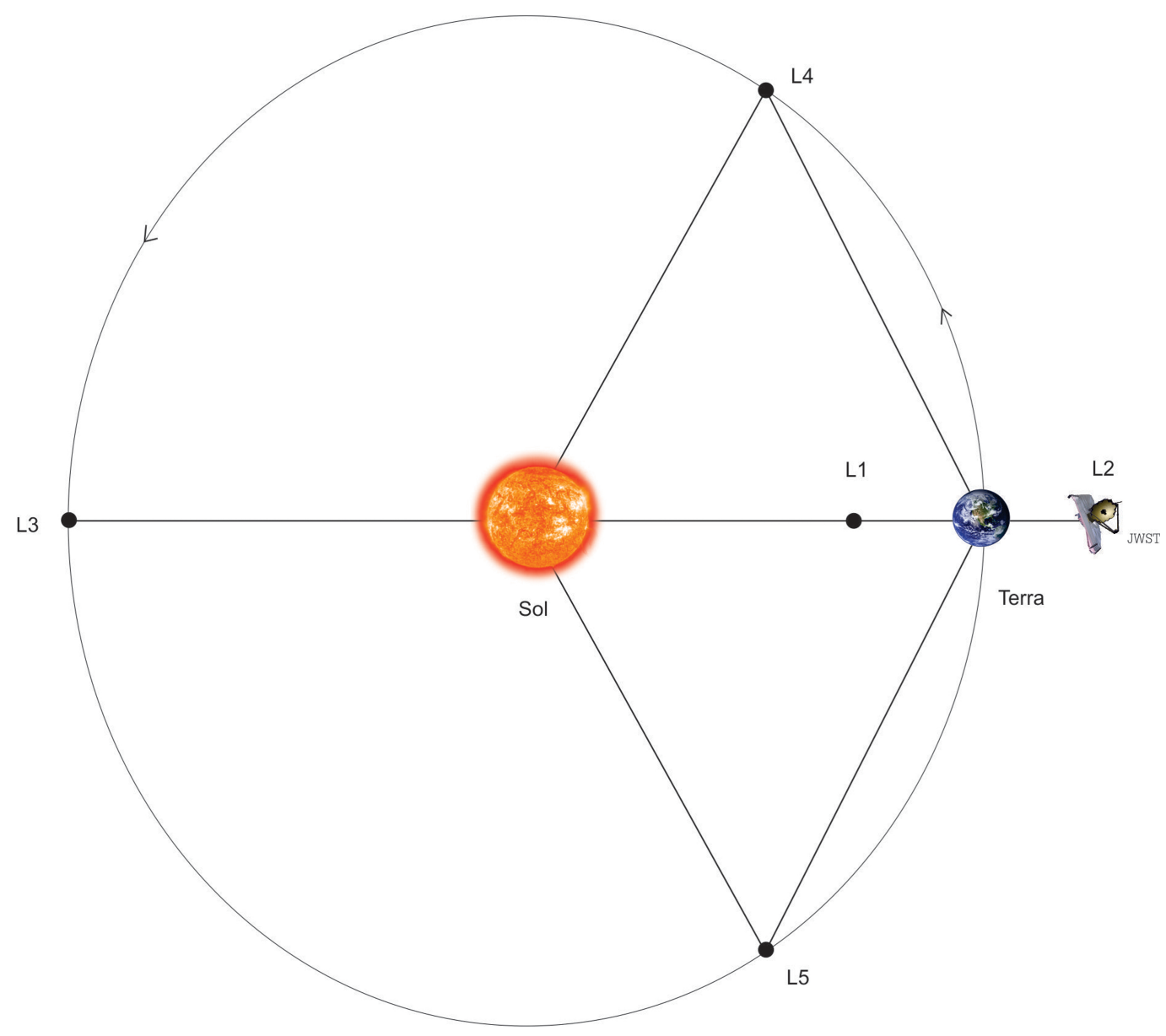

Figura 2: Figura ilustrativa mostrando a localização dos pontos de Lagrange.

Essa aproximação é válida neste caso, visto que a excentricidade da Terra é muito pequena. Fazendo com que a força centrípeta balanceie a força da gravidade $F$ teremos

$$
\frac{G M m_{s}}{(r+R)^{2}}+\frac{G m m_{s}}{R^{2}}=\frac{m_{s} v_{s}^{2}}{(r+R)}
$$

Seja a expressão para a velocidade de satelização dada por $v_{s}=2 \pi(r+R) / T_{s}$, onde nesta última expresão $T_{s}$ denota o período do satélite. Substituindo $v_{s}$ na Eq. $3 \mathrm{e}$ considerando iguais os períodos da Terra e do Satélite fazendo $T_{s}=T$, e utilizando a lei de Kepler para órbitas 


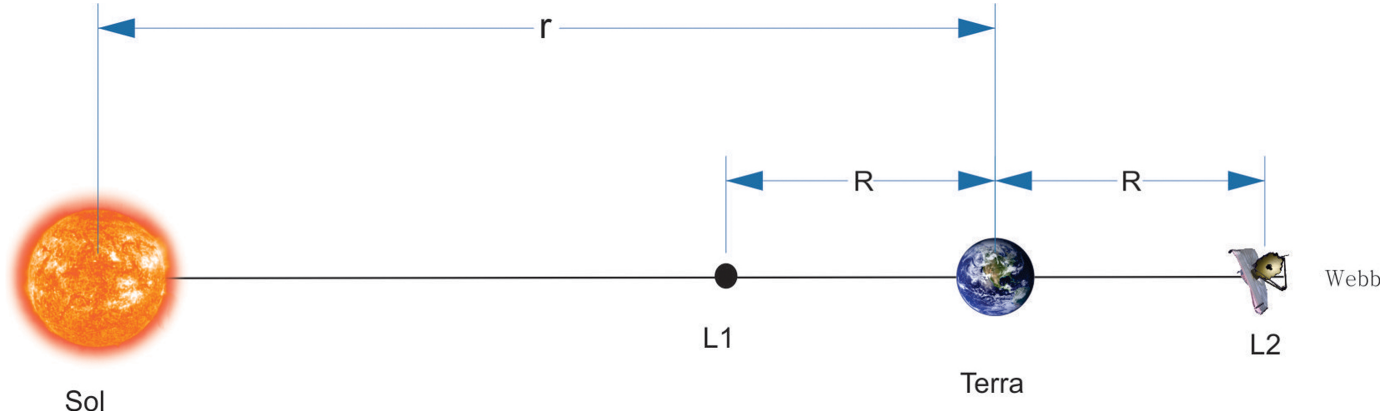

Figura 3: Ilustração das órbitas L1 and L2 considerando o sistema Sol-Terra.

circulares, fazendo $4 \pi^{2} / T^{2}=G M / r^{3}$, teremos

$$
\begin{gathered}
\frac{G M}{(r+R)}+\frac{G m(r+R)}{R^{2}}=\frac{4 \pi^{2}(r+R)^{2}}{T_{s}^{2}} \\
\frac{G M}{(r+R)^{3}}+\frac{G m}{R^{2}(r+R)}=\frac{4 \pi^{2}}{T_{s}^{2}} \\
\frac{G M}{(r+R)^{3}}+\frac{G m}{R^{2}(r+R)}=\frac{G M}{r^{3}}
\end{gathered}
$$

Dividindo ambos os lados da Eq. 5 por $G M$ e fazendo $x=m / M$ teremos

$$
\frac{1}{(r+R)^{3}}+\frac{x}{R^{2}(r+R)}=\frac{1}{r^{3}}
$$

Fazendo as manipulações apropriadas na Eq. 6 e substituindo $z=R / r$, teremos como expressão resultante

$$
\frac{1}{(1+z)^{3}}+\frac{x}{z^{2}\left(1+z^{2}\right)}=1
$$

Como $z<1$, podemos resolver a Eq. 7 utilizando o teorema binomial. Assim, com o uso de $(1 \pm z)^{n}=$ $1 \pm n z / 1 !+n(n-1) z^{2} / 2 !+\ldots$ teríamos uma aproximação em primeira ordem dada por

$$
\begin{aligned}
\frac{1}{(1+z)^{3}} & \sim 1-3 z \\
\frac{y}{z^{2}(1+z)} & \sim \frac{x}{z^{2}}(1-z)
\end{aligned}
$$

A substituição das aproximações anteriores na Eq. 7 nos leva ao seguinte resultado

$$
3 z^{3} \sim x(1-z)
$$

E tendo em vista que $z$ é muito pequeno, podemos mais uma vez fazer uma aproximação sobre este resultado e dizer que $3 z^{3} \sim x$ e logo $R \sim r \sqrt[3]{m / 3 M}$. Uma vez que $x$ é a razão entre as massas da Terra $\left(m=5,98 \times 10^{24}\right.$ $\mathrm{kg})$ e do Sol $\left(M=1,98 \times 10^{30} \mathrm{~kg}\right)$, que também é um número muito pequeno, então a razão entre as distâncias $z$ da Terra até L2 é igual a 0,01 ao longo da linha que une a Terra ao Sol. Este seria o lugar do telescópio JWST no espaço.

Podemos ainda resolver o problema da distância L2 em relação à Terra utilizando o método da esfera de Hill, um método desenvolvido por Hill em 1878. De maneira simplificada, podemos dizer que essa metodologia, em vez de de considerar as equações completas e mais complexas do movimento de três corpos, descreve o movimento da partícula em torno do corpo secundário, que neste exemplo é a Terra. A Figura 4 ilustra as Esferas de Hill.

O princípio teórico deste método contempla novamente um corpo de massa elevada e outro corpo de massa secundária, que orbita esse corpo principal. No nosso exemplo estamos considerando o Sol como corpo principal e a Terra como corpo secundário. Assim como feito anteriormente, o método da esfera de Hill também deriva das três forças, sendo que a primeira é a força da gravidade do Sol, a segunda é a força da gravidade da Terra e terceira é a força centrípeta, onde tomamos como referência o Sol e a frequência angular da Terra [21].

Conforme dito, a esfera de Hill é definida como uma esfera centrada no corpo secundário. Neste caso, o efeito combinado das três forças é direcionado para a Terra.

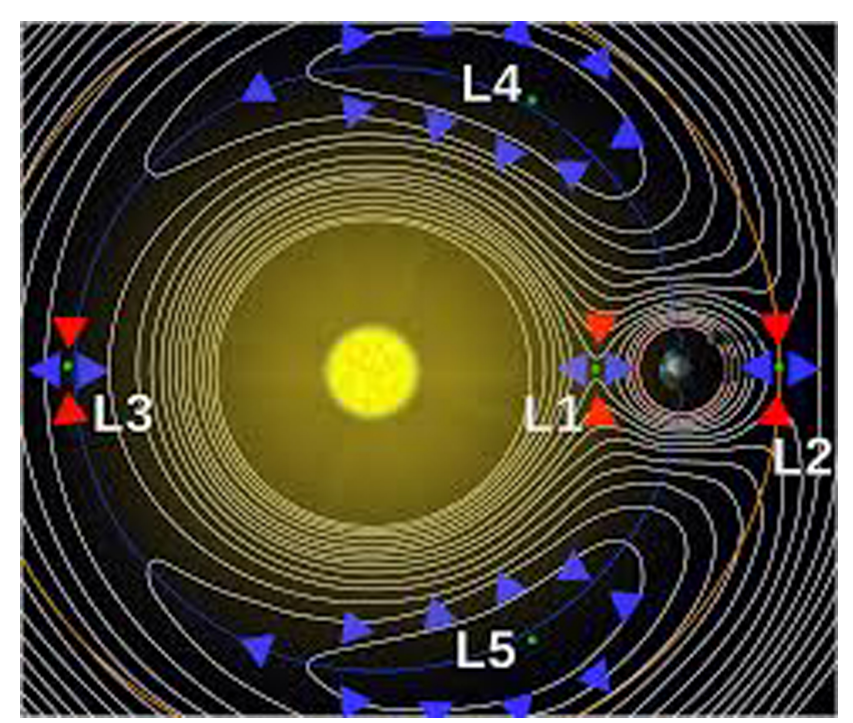

Figura 4: As esferas de Hill são as regiões circulares que envolvem as massas. Figura extraída de https://commons.wikimedia.org 
Logo, um satélite ou telescópio vinculado a este planeta permanece em sua órbita sem ser atraído para a órbita do Sol. Em termos matemáticos, a esfera de Hill estabelece uma limite ou distância máxima que um satélite pode orbitar um corpo secundário na presença de um corpo principal.

No caso do cálculo da distância L2, podemos usar o método da esfera de Hill porque a massa da Terra $(m)$ é muito menor que a massa do Sol $(M)$, logo tanto L1 como L2 estão aproximadamente a distâncias iguais $R$ em uma aproximação de primeira ordem do menor objeto, que será igual ao raio da esfera de Hill dado por

$$
R=r \sqrt[3]{\frac{m}{3 M}}
$$

Maiores detalhes sobre este cálculo podem ser encontrados em 21]. Este resultado coincide com a expressão obtida anteriormente utilizando a aproximação binomial. Neste caso, a razão $z=R / r$ para o cálculo de L2 é $z=0,01$ e assim, numericamente, o cálculo através da esfera de Hill para a localização dos pontos de equilíbrio coincide com a aproximação dada pelo teorema binomial. Poderíamos também reescrever a expressão com resultado da esfera de Hill assim

$$
z=\sqrt[3]{\frac{m}{3 M}}=\alpha
$$

Como a razão $z=R / r$ é pequena, existe uma solução próxima de $z=\alpha$, onde $\alpha$ é um parâmetro frequentemente usado na Equações de Hill que envolvem os demais pontos L1, L3, L4 e L5. Neste caso, o método de Hill também possibilita expressar $z$ como funções de séries de $\alpha$, e assim teríamos como resultado para a localização de L2 21].

$$
z=\alpha+\frac{1}{3} \alpha^{2}-\frac{1}{9} \alpha^{3}-\frac{31}{81} \alpha^{4}+O\left(\alpha^{5}\right)
$$

Fazendo as substituições das massas da Terra e do Sol, obteríamos $z=0,010055764$ utilizando as séries em $\alpha$.

A Terra descreve uma órbita praticamente circular em torno do Sol, mas esse não é o caso de Mercúrio, que tem a maior excentricidade do sistema solar $(e=0,206)$ depois de Plutão $(e=0,248)$, que não é mais considerado um planeta desde 2006. Se fizermos o cálculo para a posição L2 para planetas descrevendo órbitas com excentricidade não nula, é necessário usar a elipse como referência. Em coordenadas polares, uma das maneiras de descrever a posição da elipse, é considerando a origem em um dos seus focos. Podemos adotar essa descrição para generalizar o resultado obtido. Sendo assim, no caso de uma órbita elíptica, teríamos a variável $r$ sendo expressa como

$$
r=\frac{a\left(1-e^{2}\right)}{1+e \cos \theta}
$$

onde $a$ denota um dos semi-eixos da elipse, e $e$ denota a excentricidade da mesma. Se substituirmos $r$ na Eq 9 considerando este igual a distância do periélio ao foco (Sol), ou seja $r=a(1-e)$, teremos

$$
R=a(1-e) \sqrt[3]{\frac{m}{3 M}}
$$

Este resultado é a generalização dos cálculos anteriores para casos de órbitas elípticas.

Além do clássico método das séries e do método da esfera de Hill, muito utilizado em estudos do sistema solar, com a evolução dos grandes computadores, pode-se solucionar problemas de três corpos utilizando-se a computação numérica, fazendo por exemplo a parametrização das equações de movimento em matrizes, utilizando-se métodos como o de Runge-Kutta (RKF4) entre outros. Outra maneira de se determinar a localização de L2 se dá através do cálculo numérico. Podemos melhorar o resultado obtido na aproximação binomial para $z$ na Eq.8. Para isso, dentre alguns métodos métodos numéricos, vamos utilizar o método de Newton-Raphson.

O método de Newton-Raphson estima numericamente as raízes de uma função escolhendo-se uma aproximação inicial para o valor de $z$ na Eq 8 Seja $z_{0}=0,01$ uma boa estimativa de $z$ e portanto $z=z_{0}+h, \operatorname{logo} h=z-z_{0}$ e neste caso $h$ mensura quão distante $z_{0}$ está da solução. Como $h$ é pequeno e denota uma pequena correção no valor de $z$, conclui-se que

$$
0=f(z)=f\left(z_{0}+h\right) \approx f\left(z_{0}\right)+h f^{\prime}\left(z_{0}\right)
$$

Logo $h \approx-f\left(z_{0}\right) / f^{\prime}\left(z_{0}\right)$ e $\operatorname{assim} z=z_{0}-f\left(z_{0}\right) / f^{\prime}\left(z_{0}\right)$. Uma nova estimativa do valor procurado $z_{1}$ é dada por

$$
z_{1}=z_{0}-\frac{f\left(z_{0}\right)}{f^{\prime}\left(z_{0}\right)}
$$

A estimativa consecutiva envolvendo o valor procurado $z_{2}$ é obtida do mesmo modo que $z_{1}$ foi obtido de $z_{0}$ e assim por diante, de modo que $z_{2}=z_{1}-f\left(z_{1}\right) / f^{\prime}\left(z_{1}\right)$. Podemos concluir então que

$$
z_{n+1}=z_{n}-\frac{f\left(z_{n}\right)}{f^{\prime}\left(z_{n}\right)}
$$

Com o uso deste método e utilizando a aproximação inicial de $z_{0}=0,01$, obtivemos que $z=0,01000559686$.

\section{Conclusão}

Neste trabalho fizemos um estudo sobre o futuro telescópio JWST com ênfase no cálculo do posicionamento deste no espaço. Este problema enquadra-se no problema restrito de três corpos, onde uma das massas é desprezível em relação as outras duas massas maiores (Sol e Terra). Além disso, fizemos uso da lei da gravitação de Newton para estudar as forças envolvidas na estimativa da posição L2 do telescópio JWST. Sendo assim, o problema aqui descrito foi tratado de um ponto de vista clássico, visto que assumimos que os corpos são objetos esféricos 
e pontuais, descritos pela gravitação de Newton. Para estimativa da posição L2, utilizamos o método das séries ou teorema Binomial e o método da Esfera de Hill. Além disso, descrevemos uma possível aplicação numérica para este cálculo através da aplicação do método de Newton Raphson para melhorar o resultado obtido.

\section{Referências}

[1] https://www.nasa.gov/feature/nasa-s-james-webbspace-telescope-to-be-launched-spring-2019.

[2] https://www.nasa.gov/content/goddard/2017/ highlights-of-hubble-s-exploration-of-theuniverse

[3] https://keplerscience.arc.nasa.gov/

[4] J.P. Gardner and the JWST Science Working Group, American Astronomical Society 41, 254 (2009).

[5] J.P. Gardner, S. Massimo, L. Mather and JWST Science Working Group, James Webb Space Telescope, Studies of Dark Energy (White Papers NASA, Washington, DC, 2010).

[6] F.L. Silveira, L.F. Braun e T. Braun, Rev. Bras. Ensino Fís. 32, 1 (2010).

[7] G. Taubes, Science 283, 620 (1999).

[8] H. Stockman, S. Space Telescope Science Institute, NGST Study Team, The Association of Universities for Research in Astronomy, Inc, v. 1, 263 (1997).

[9] W. Lambright, Powering Apollo (Johns Hopkins University Press, Baltimore, 1995), v. 1, p. 2.

[10] J. Norwood, H. Hammel, S. Milam, J. Stansberry, J. Lunine, N. Chanover, D. Hines, G. Sonneborn, M. Tiscareno, M. Brown and P. Ferruit, Solar System Observations with the James Webb Space Telescope (Cornell University Library, New York 2014), v. 2.

[11] R. Farquhar, D. Muhonen and L.C. Church, Journal of the Astronautical Sciences 33, 235 (1985).

[12] C. Reynolds, Nature 438, 32 (2005).

[13] V. Domingo, B. Fleck and A.I. Poland, Solar Physics 162, 1 (1995).

[14] L. Phillips, Nature 491, 330 (2012).

[15] F.P.J. Valero, Science 3, 775 (2006).

[16] M.E. Gomez, S. Lola, P. Naranjo and J. RodriguezQuintero, High Energy Physics 43, 7 (2009).

[17] C. Li, J. Liu, X. Ren, W. Zuo, X. Tan, et al., Space Science Reviews 190, 85 (2015).

[18] L. Aleman, T. Ueta, D. Ladjal, K.M. Exter, J.H. Kastner, et al., Journal: A \& A. 565, 79 (2014).

[19] S. B. Broschart, T.H. Sweetser, V. Angelopoulos, D. Folta and M. Woodard, (Preprint) AAS 11-509, (2013), disponível em http://www.igpp.ucla.edu/public/ THEMIS/SCI/Pubs/artemis/2011_non-refereed/ Broschart_etal_ArtemisLunarOrbits_2011.pdf

[20] Z.E. Musielak and B. Quarles, Reports on Progress in Physics 77, 3 (2014).

[21] C.D. Murray and S. F. Dermott, Solar System Dynamics (Cambridge University Press, Cambrigde, 1999) 1, p. 4.

[22] J. Barrow-Green, American Mathematical Society and London Mathematical Society 11, 7 (1997).

[23] K.H. Stevanovic, Scientific Technical Review 7, 4 (2007). 